\title{
Variação da regulagem no perfil transversal de aplicação com distribuidores centrífugos
}

\author{
Variation in the adjustment of an application transversal profile with centrifugal broadcaster spreaders
}

\author{
Isaias Salim Farret ${ }^{*}$ José Fernando Schlosser $^{\mathrm{I}}$ Reges Durigon $^{\mathrm{I}}$ Valmir Werner ${ }^{\mathrm{II}}$ \\ Marcelino Knob ${ }^{\text {II }}$
}

\section{RESUMO}

Os distribuidores centrífugos revolucionaram a tecnologia de aplicação de produtos sólidos na agricultura por apresentarem grande capacidade de campo operacional e pela grande amplitude de dosagens que consegue aplicar. No entanto, este equipamento apresenta alguns inconvenientes, como a dificuldade em aplicar homogeneamente o material na largura de trabalho utilizada, mesmo com uma aparente facilidade de regulagem. O objetivo deste trabalho foi comparar tratamentos relacionados a regulagens operacionais buscando equilibrar e uniformizar o perfil transversal de distribuição, com a maior capacidade de campo operacional, para os diferentes produtos sólidos. A máquina avaliada foi um distribuidor centrífugo de produtos sólidos bidisco. Os resultados nos levam a concluir que as velocidades média e alta da esteira transportadora para a distribuição de calcário e a velocidade baixa para a distribuição de aveia fornecem uma ampla e suficiente faixa de dosagens com a variação de abertura da comporta dosadora. O aumento da vazão e do adiantamento da posição das aletas possibilita maior capacidade de campo operacional ao conjunto mecanizado, pela possibilidade de operar com maior largura útil de aplicação e maior velocidade de deslocamento. A largura máxima recomendada de aplicação foi de 13,5 metros para o calcário e para a aveia.

Palavras-chave: distribuidor a lanço, uniformidade de aplicação, coeficiente de variação.

\section{ABSTRACT}

Centrifugal broadcaster spreaders have been a breakthrough technology in the application of solid materials in agriculture because they have a high performance in field operations and are able to apply a broad range of rates. However, this equipment has some disadvantages, such as the difficulty in homogenously applying the material although adjusting is a relatively simple task. The objective of this study was to compare treatments of operational adjustments in order to balance and uniformize the transversal pattern of distribution, aiming a higher field operational capacity, for different solid products. The machine evaluated was a centrifugal broadcaster for solid material. Results showed that medium and high speeds of the conveying chain for lime distribution and the low speed for oat distribution give a large and enough sufficient range of application rate by varying the opening of the metering device. Increasing the flow and advancing the impellers position gave a higher field operational capacity because of the possibility of a larger effective width as well as a higher travel speed. The maximum application width recommended is 13.5 meters for both lime and oat.

Key words: broadcaster spreaders, uniformity of distribution, coefficient of variation.

\section{INTRODUÇÃO}

Os distribuidores centrífugos são equipamentos que revolucionaram a tecnologia de aplicação de produtos sólidos na agricultura por suas características de simplicidade construtiva, facilidade de regulagem e alta capacidade operacional. O seu principal uso é na aplicação de produtos agrícolas de correção e adubação do solo como calcário, gesso agrícola, adubo mineral, uréia e na semeadura a lanço de pastagens como da aveia e azevém.

Estes equipamentos utilizam a energia gerada pela tomada de potência (TDP) do trator para

IDepartamento de Engenharia Rural, Centro de Ciências Rurais (CCR), Universidade Federal de Santa Maria (UFSM), 97105-900

Santa Maria, RS, Brasil. E-mail: ifarret@uol.com.br. *Autor para correspondência.

IIPrograma de Pós-graduação em Engenharia Agrícola, CCR, UFSM, Santa Maria, RS, Brasil. 
imprimirem energia às partículas e conseqüentemente lançá-las a distâncias consideráveis. A maioria utiliza disco como elemento lançador, valendo-se da força centrífuga para imprimir energia às partículas que chegarão ao solo, desenvolvendo uma trajetória balística. Dessa forma, os produtos mais leves, como sementes, serão lançados a menores distâncias e os mais pesados, como uréia e fertilizantes, a distâncias maiores, determinando maior largura de aplicação.

As principais características dos discos que afetam a distribuição dos produtos são o formato e o ângulo das aletas e o diâmetro, a rotação e a altura do disco. Segundo BERNACKI \& KANAFOJSKI (1972), quando as aletas são posicionadas alternadamente, o aumento no seu ângulo negativo de colocação diminui a largura de trabalho do distribuidor. Por outro lado, um aumento no ângulo positivo afeta a qualidade de distribuição. Para SOUZA (1984), um distribuidor com aletas em posição alternada, com dois valores de inclinação e posicionada com o mesmo valor, poderiam impulsionar velocidades divergentes ao fertilizante.

Quanto à altura, ao diâmetro e às rotações do disco distribuidor, estudos de BERNACKI \& KANAFOJSKI (1972) mostram que os discos não devem trabalhar em alturas superiores a $0,80 \mathrm{~m}$ do solo, ter diâmetro entre 0,50 a 0,60m e uma rotação de 540 a 700rpm, dando assim uma velocidade periférica de 6 a $14 \mathrm{~m} . \mathrm{s}^{-1}$. Nesse sentido, SILVA (1982) verificou que a queda da rotação do disco causou uma diminuição da largura da faixa, concentrando a deposição do fertilizante nas proximidades do disco, devido à redução da velocidade tangencial e, conseqüentemente, das partículas ao abandonarem as aletas.

O método padronizado para avaliar o trabalho destes distribuidores, conforme ASAE (1995), está baseado em norma oficial. Esta norma define termos, estabelece as condições e os procedimentos de ensaio, assim como a maneira de relatar os resultados desta avaliação. Ela é semelhante à norma ISO 5690 (ISO, 1982), que igualmente trata de condições de ensaio, procedimentos, resultados esperados.

$\mathrm{Na}$ avaliação do desempenho de um distribuidor centrífugo, um dos critérios mais importante é a uniformidade de distribuição (SRIVASTAVA et al., 1993). Segundo MÁRQUEZ (2001), a uniformidade de distribuição de produtos sólidos é influenciada por diversos fatores, entre eles, a forma construtiva do espalhador e a sobreposição entre as passadas. A distância horizontal pela qual as partículas são lançadas é afetada pelo tamanho de partícula, densidade, forma, padronização e velocidade de rotação dos discos. Os componentes de uma mistura seca tendem a separarse, sendo projetadas mais longe as partículas maiores e de mesma densidade. O vento também afeta a distância e, conseqüentemente, o padrão de distribuição.

Para obter uniformidade de trabalho desejável de um distribuidor centrífugo, é necessário determinar o diagrama de distribuição e os valores correspondentes à largura máxima e à largura útil de trabalho (ORTIZ-CAÑAVATE et al., 1989). O coeficiente de variação é um dos parâmetros utilizados para avaliar a uniformidade de distribuição. Para VALDEZ (1978), um perfil de distribuição com coeficiente de variação de 33\% é suficientemente uniforme, que não chega a afetar significativamente o rendimento da cultura. Também ORTIZ-CAÑAVATE et al. (1989) estima que coeficientes de variação de aproximadamente $20 \%$ são satisfatórios para adubos granulados e 30\% para materiais pulverulentos.

Por outro lado, considera-se como grau de uniformidade de distribuição muito bom um coeficiente de variação até $10 \%$, bom até $20 \%$, regular até 33\% e pobre acima de 33\% (WEISS, 1986). Em acordo, DALLMAYER (1985) relata que a Sociedade de Agricultura Alemã não reconhece distribuidores centrífugos que apresentam um perfil transversal com valores de coeficiente de variação superior a 12,5\%.

Este trabalho teve como objetivo geral comparar tratamentos que uniformizem o perfil transversal de distribuição com um distribuidor a lanço centrífugo, combinado com a maior capacidade de campo efetiva possível, por meio de regulagens operacionais para os diferentes produtos sólidos.

Especificamente, buscou-se identificar as regulagens operacionais mais adequadas à aplicação dos diferentes produtos sólidos, determinando o coeficiente de variação no perfil transversal e a largura útil de aplicação com o distribuidor centrífugo.

\section{MATERIAL E MÉTODOS}

A máquina avaliada foi um distribuidor centrífugo novo, bidisco, marca Boelter, tipo Floater 10 - Plantio Direto, modelo DC 10.000. As especificações técnicas do distribuidor centrífugo estão descritas na tabela 1.

As avaliações foram desenvolvidas de acordo com a Norma ISO 5690 (ISO, 1982), como preconizado por MIALHE, (1996). Esta norma estabelece os critérios para os ensaios estáticos e dinâmicos. Os ensaios foram efetivados em condições meteorológicas satisfatórias, como ausência de chuva, umidade relativa do ar inferior a $80 \%$ e velocidade máxima do vento inferior a $2 \mathrm{~m} \mathrm{~s}^{-1}\left(7,2 \mathrm{~km} \mathrm{~h}^{-1}\right)$, não sendo toleradas rajadas de vento. 
Tabela 1 - Especificações técnicas do distribuidor centrífugo.

\begin{tabular}{lllll}
\hline & Dimensões gerais & \multicolumn{2}{c}{ Mecanismo de dosagem } & \multicolumn{2}{c}{ Mecanismo de distribuição } \\
Largura & $2100 \mathrm{~mm}$ & Largura comporta & $505 \mathrm{~mm}$ & $\mathrm{~N}^{\circ}$ discos/aletas \\
\hline Altura & $2300 \mathrm{~mm}$ & Largura da esteira & $510 \mathrm{~mm}$ & Regulagem das aletas \\
Comprimento & $4770 \mathrm{~mm}$ & Rotação dos discos & $550 \mathrm{rpm}$ & Altura dos discos \\
Massa & $1523 \mathrm{~kg}$ & Rotação esteira 1 & 3,5rpm & Distância entre discos \\
Pneus & Super Flot & Rotação esteira 2 & 20rpm & Diâmetro discos \\
Bitola & $500 / 60-22,5$ & Rotação esteira 3 & 43rpm & Acionamento \\
\hline
\end{tabular}

${ }^{1}$ At - atrasada; Ad - adiantada; $\mathrm{R}$ - radial.

Neste trabalho foi proposto um estudo do efeito da combinação entre três fatores sobre a uniformidade de distribuição transversal do produto aplicado e sobre a largura da faixa de aplicação, sendo os fatores: produtos, regulagem da comporta e posição das aletas; com os seguintes níveis: calcário e aveia para o primeiro fator, posições um, dois, seis, oito e 15 para o segundo fator e radial, adiantada e atrasada para o terceiro fator.

Realizou-se, em laboratório, um ensaio estático de recolhimento das doses aplicadas (kg.min ${ }^{-1}$ ) para cada produto analisado. Com o auxílio de recipientes coletores e uma lona, foram coletadas as quantidades de produto aplicadas na rotação padrão da TDP (540rpm), em 30 ou 60 segundos para cada posição da alavanca de abertura do mecanismo dosador. Foi determinada a vazão de cada produto em todas as possíveis regulagens da máquina. Com as vazões (kg $\mathrm{min}^{-1}$ ) obtidas, utilizando os valores projetados para larguras de trabalho (10, 12 e 14 metros) e para velocidade de deslocamento do equipamento ( 4 a $10 \mathrm{~km}$ $\mathrm{h}^{-1}$ ), foi possível calcular as quantidades de cada produto que poderá ser aplicada por unidade de área $\left(\mathrm{kg} \mathrm{ha}^{-1}\right)$.

Para a avaliação dos perfis transversal e longitudinal de distribuição dos produtos, foram escolhidas vazões próximas à recomendada agronomicamente para cada produto analisado. A coleta dos produtos foi realizada em bandejas padronizadas, com dimensões de $500 \mathrm{~mm}$ de lado e $150 \mathrm{~mm}$ de profundidade. Nos ensaios foi utilizado o trator da marca Massey Ferguson, modelo 5310, do tipo 4x2 com tração dianteira auxiliar (TDA). A rotação de trabalho do motor foi de 2030rpm, que proporcionou 540rpm na TDP e velocidade de deslocamento de $7,4 \mathrm{~km} \mathrm{~h}^{-1}$ em $3^{\text {a }}$ marcha do regime reduzido e velocidade alta. No ensaio deste equipamento, foram realizadas variações de regulagens da velocidade da esteira alimentadora de produto (baixa, média e alta), abertura da comporta dosadora e posição de aletas (radial, atrasada e adiantada).

Para o ensaio do perfil transversal, as bandejas foram distribuídas transversalmente à passagem do conjunto mecanizado, na largura total de trabalho. Foram utilizadas 54 bandejas, 27 para cada lado, com o eixo central transversal estando em acordo com o eixo de deslocamento do conjunto. Para a elaboração dos histogramas de aplicação, determinação do coeficiente de variação e largura de trabalho, foi utilizado o programa informático Adulanço, versão 2.0.14 (MOLIN et al., 1992).

Os histogramas representativos do perfil permitem a visualização da distribuição de produto em toda a largura da faixa de deposição sem a contribuição da sobreposição de passadas adjacentes. O eixo das abscissas representa a posição de cada bandeja que recebeu deposição de produto, na mesma ordem/ posição em que estiveram dispostas na pista no momento da amostragem, porém, numeradas seqüencialmente da esquerda para a direita. No eixo das ordenadas, foram representadas as quantidades (em gramas) que cada bandeja recebeu.

Por outro lado, os gráficos do Coeficiente de Variação quantificam as variações de volume do produto depositado em toda a largura da faixa de distribuição, neste caso, com a contribuição da sobreposição de passadas adjacentes, segundo o sistema de operação contínuo. O eixo das abscissas representa a largura da faixa de aplicação calculada para cada CV correspondente no eixo das ordenadas.

\section{RESULTADOS E DISCUSSÃO}

As vazões de calcário e aveia determinadas em laboratório (Figura 1) permitem simular diferentes doses de aplicação. A dose aplicada por área $\left(\mathrm{kg} \mathrm{ha}^{-1}\right)$ para determinada velocidade e largura de trabalho é definida em função de dois parâmetros: velocidade da

Ciência Rural, v.38, n.7, out, 2008. 


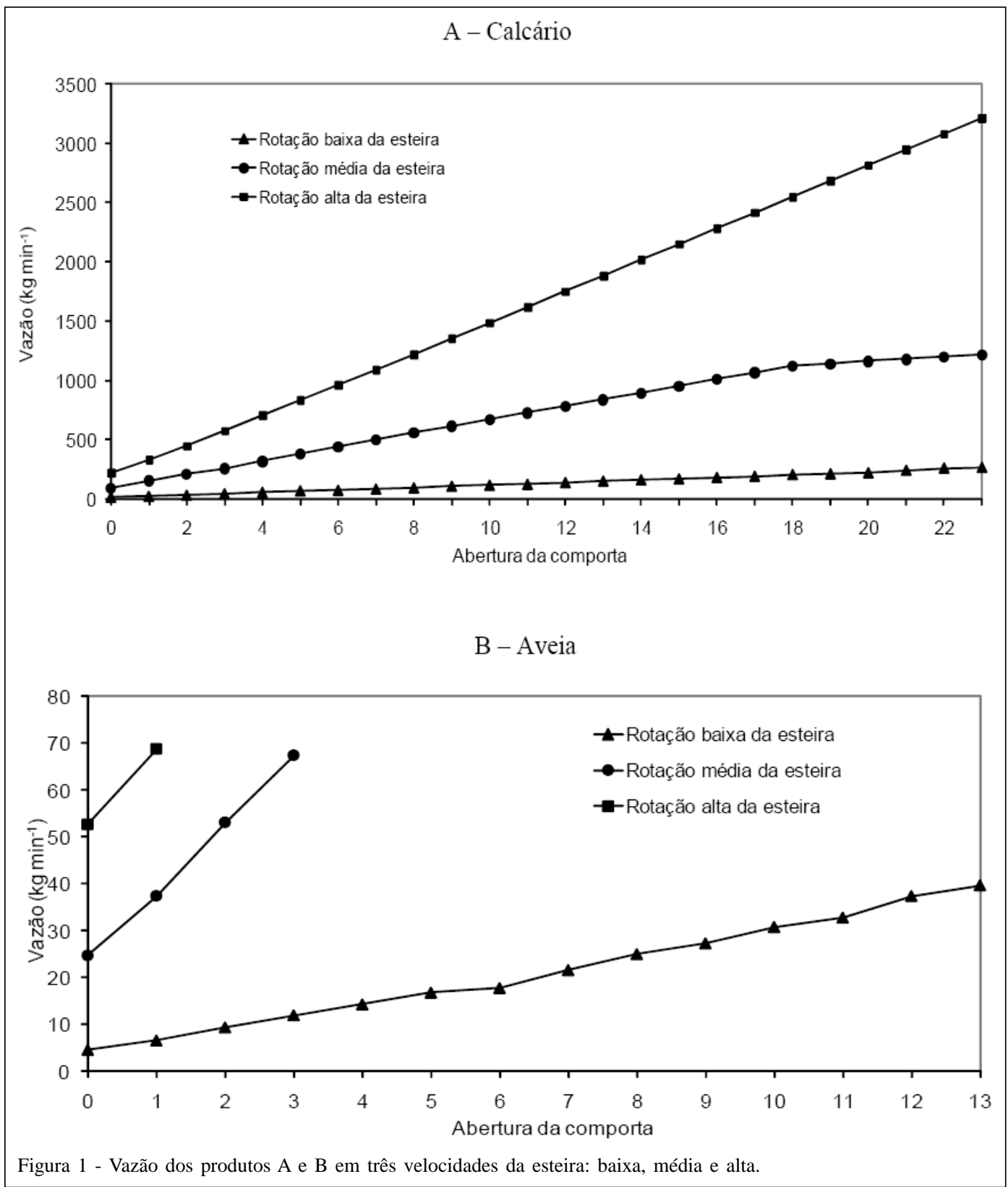

esteira e abertura da comporta. Para aplicações a $8 \mathrm{~km} \cdot \mathrm{h}^{-1}$ e largura de 12 metros, nas velocidades baixa, média e alta da esteira, pode-se aplicar taxas entre $101 \mathrm{e} 1655 \mathrm{~kg}$ $\mathrm{ha}^{-1}, 586$ e $7.615 \mathrm{~kg} \mathrm{ha}^{-1}$ e 1.375 e $20.098 \mathrm{~kg} \mathrm{ha}^{-1}$, respectivamente. Dessa forma, para a utilização do equipamento na aplicação de calcário, recomendam-se as velocidades de esteira média e alta, pois a velocidade baixa somente permite a aplicação em dosagens que, normalmente, não são utilizadas para este tipo de produto.

Para a utilização do distribuidor centrífugo na semeadura de aveia, deve-se operar a esteira transportadora na velocidade baixa, a qual fornece uma grande amplitude de vazão (Figura 1B) somente com a variação da abertura da comporta. Com base nos valores obtidos para vazão $\left(\mathrm{kg} \mathrm{min}^{-1}\right)$ de aveia e fixandose a largura de trabalho em 12 metros e a velocidade de deslocamento em $8 \mathrm{~km} \cdot \mathrm{h}^{-1}$, a regulagem da comporta permite aplicar doses entre 28 e $247 \mathrm{~kg} \mathrm{ha}^{-1}$.

Analisando o comportamento do perfil transversal de distribuição de calcário, em função da variação da posição das aletas no disco (Figura 2), verifica-se que não houve influência dessa regulagem sobre o perfil transversal de distribuição do calcário. 


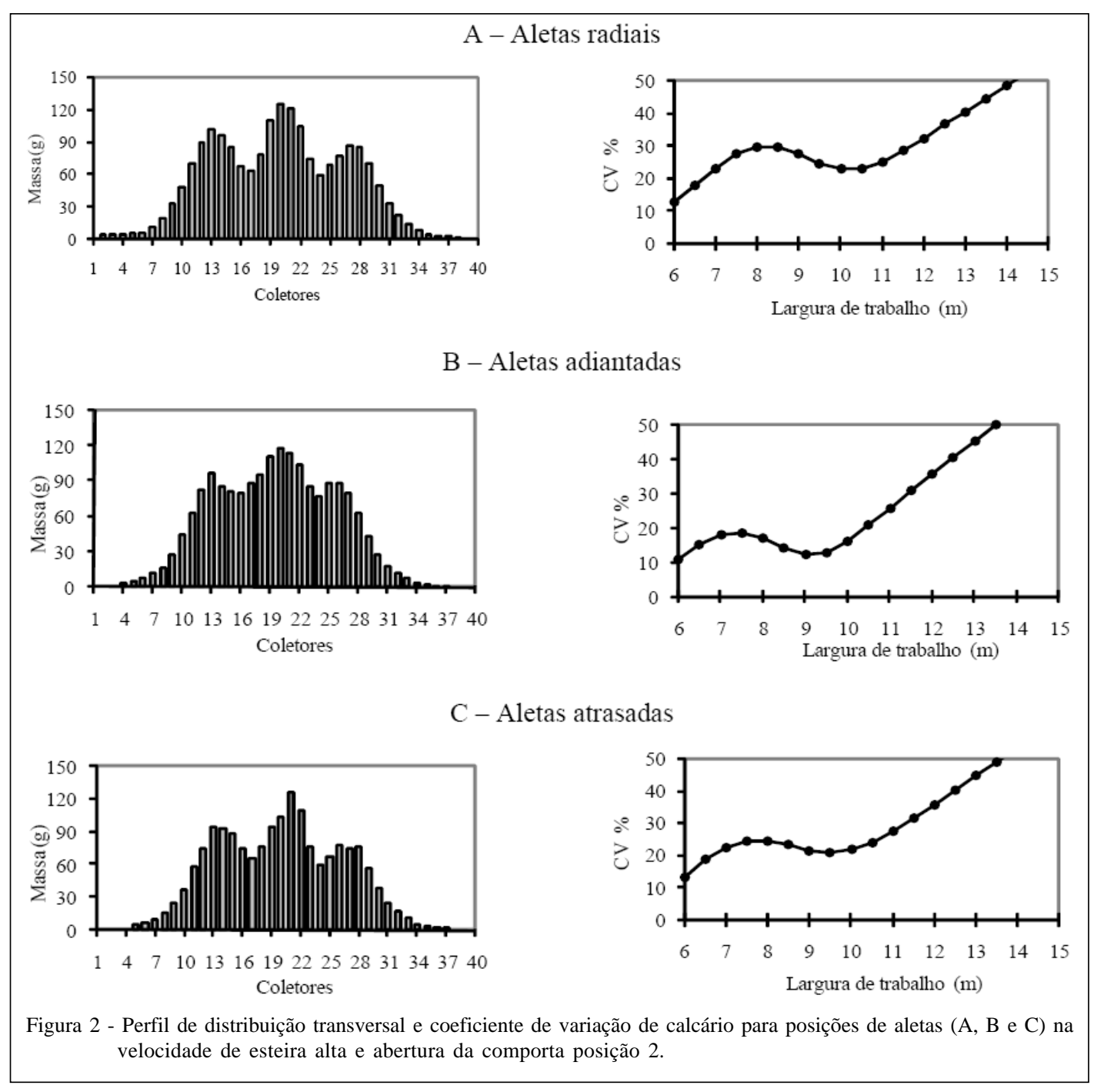

Para a aveia (Figura 3), as posições de aleta radial e atrasada determinaram um perfil transversal com maior deposição de produto na faixa central de deslocamento do conjunto mecanizado, enquanto que a posição adiantada de aleta determinou uma deposição de produto mais equilibrada ao longo do perfil transversal. Este comportamento pode ser explicado pelo fato de que as partículas maiores e de maior densidade são lançadas a maiores distâncias - respondem mais ao aumento de inércia e à direção de lançamento determinados pelo avanço da aleta - característica que a aveia apresenta devido ao enchimento de grãos. Analisando o perfil de distribuição transversal para ambos os produtos avaliados, observa-se uma maior quantidade de produto recolhida ao longo do eixo de deslocamento do conjunto mecanizado. Isso pode estar relacionado com o acúmulo de produto na parte frontal da esteira transportadora que, a partir de determinado volume de produto acumulado, cai nas bandejas centrais. Este acúmulo de produto resulta do transporte de material nas travessas da esteira durante o retorno da mesma e é proporcional à velocidade da esteira, sendo verificado tanto para calcário quanto para aveia.

A posição atrasada das aletas não se mostrou adequada para distribuição de aveia em nenhuma das larguras experimentadas, pois apresentou altos coeficientes de variação em relação aos padrões utilizados neste experimento e recomendados por VALDEZ (1978) e por ORTIZ-CAÑAVATE et al. (1989).

Para melhor avaliação da regularidade da distribuição transversal dos produtos pelo distribuidor avaliado, apresenta-se a tabela 2, que reúne os valores dos Coeficientes de Variação resultantes das combinações de regulagens analisadas neste experimento para 10, 12 e 14 metros de largura da faixa

Ciência Rural, v.38, n.7, out, 2008. 

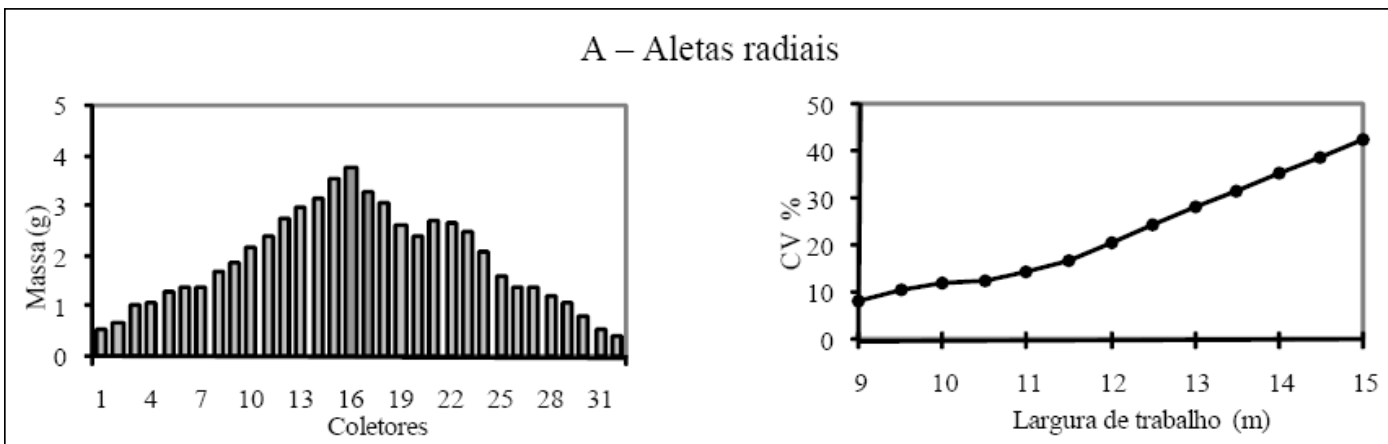

B - Aletas adiantadas
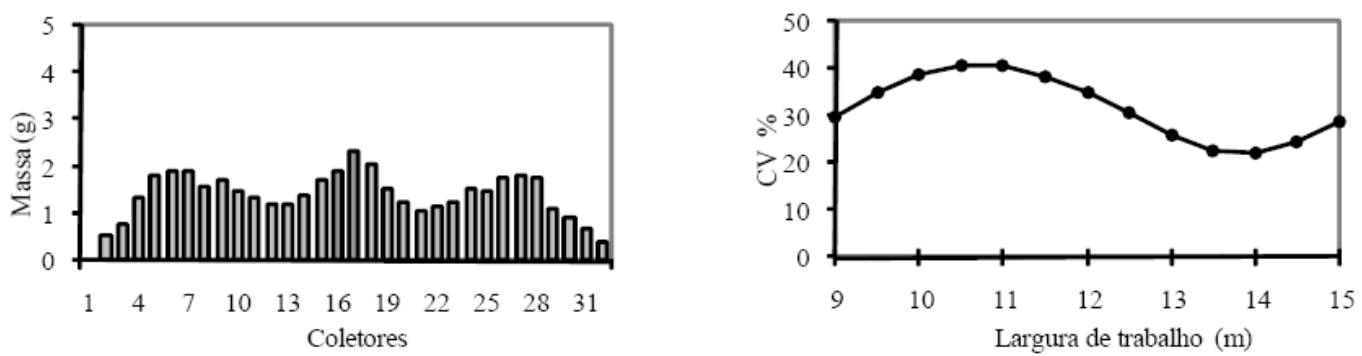

C - Aletas atrasadas
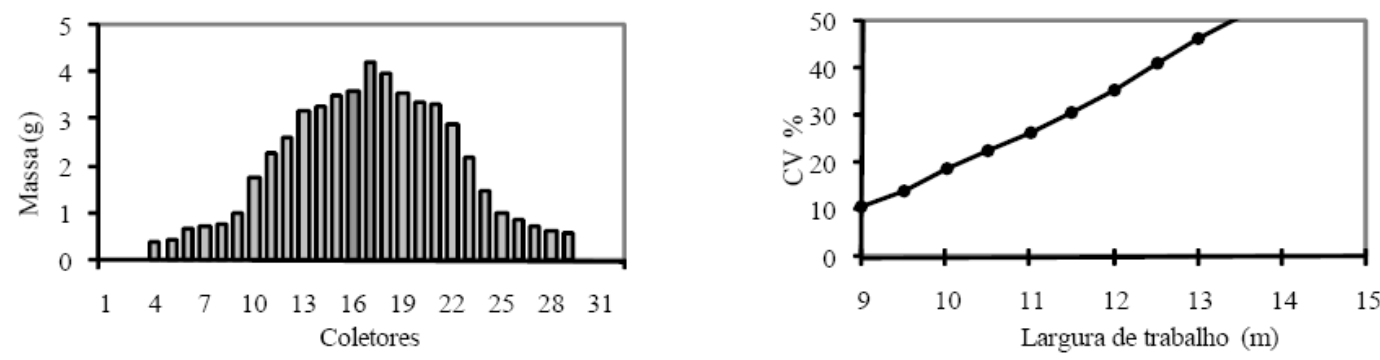

Figura 3 - Perfil de distribuição transversa e coeficiente de variação de aveia para posições de aletas (A, B e C) com velocidade de esteira baixa e abertura da comporta na posição 3.

de aplicação, nos sistemas contínuo e alternado. Ainda, com o objetivo de facilitar a visualização do comportamento do Coeficiente de Variação nas diferentes condições, os valores considerados dentro dos limites aceitáveis foram destacados em negrito. $\mathrm{Na}$ avaliação da regularidade de distribuição transversal deste trabalho, com base na revisão bibliográfica, foi considerado como limite máximo aceitável o Coeficiente de Variação de 33\% para o calcário e de 20\% para aveia.

Para aplicação de calcário com utilização do distribuidor avaliado, as velocidades média e alta da esteira fornecem uma ampla e suficiente faixa de dosagens somente com a variação da abertura da comporta dosadora do produto. Para semeadura de aveia com utilização do distribuidor avaliado, a velocidade baixa da esteira fornece suficientes possibilidades de dosagens somente com a variação da abertura da comporta dosadora do produto.

Das condições analisadas, a que combina abertura de comporta 15, posição radial das aletas, largura útil de 10 metros e sistema de aplicação alternado apresentou a melhor uniformidade de distribuição de calcário e a que combina abertura de comporta 1, posição radial das aletas, largura útil de 10 metros e sistema de aplicação alternado apresentou a melhor uniformidade de distribuição de aveia.

A maior largura de aplicação de calcário com o distribuidor avaliado foi 13 metros, considerando como aceitável o Coeficiente de Variação de até 33\% e a maior largura de aplicação de aveia com o distribuidor avaliado foi 13,5 metros, considerando como aceitável o Coeficiente de Variação de até 20\%. 
Tabela 2 - Coeficientes de variação (\%) para diferentes larguras de trabalho com distribuidor centrífugo em diferentes sistemas de trabalho.

\begin{tabular}{|c|c|c|c|c|c|c|c|c|c|c|c|}
\hline \multicolumn{3}{|c|}{----------------Tratamento--------------- } & \multicolumn{3}{|c|}{----------10m---------- } & \multicolumn{3}{|c|}{----------12m---------- } & \multicolumn{3}{|c|}{----------14m---------- } \\
\hline Produto & Regulagem & Aleta & $\mathrm{C}$ & $\mathrm{AE}$ & $\mathrm{AD}$ & $\mathrm{C}$ & $\mathrm{AE}$ & $\mathrm{AD}$ & $\mathrm{C}$ & $\mathrm{AE}$ & $\mathrm{AD}$ \\
\hline Calcário & 2 & Radial & 21,7 & 18,6 & 32,0 & 35,6 & 22,1 & 48,8 & 53,3 & 40,7 & 65,0 \\
\hline Calcário & 2 & Adiantada & 22,8 & 21,5 & 23,4 & 32,3 & 32,7 & 31,6 & 48,4 & 48,1 & 48,2 \\
\hline Calcário & 2 & Atrasada & 16,2 & 12,2 & 26,3 & 35,7 & 25,0 & 47,3 & 54,2 & 43,6 & 64,7 \\
\hline Calcário & 8 & Radial & 21,7 & 22,4 & 20,0 & 20,7 & 22,2 & 18,2 & 36,8 & 39,3 & 33,9 \\
\hline Calcário & 15 & Radial & 12,0 & 10,4 & 9,8 & 27,6 & 28,0 & 26,8 & 43,4 & 43,5 & 42,7 \\
\hline Aveia & 1 & Radial & 13,6 & 9,6 & 13,6 & 24,6 & 21,1 & 25,9 & 37,3 & 37,4 & 36,2 \\
\hline Aveia & 3 & Radial & 12,3 & 10,5 & 12,4 & 20,8 & 22,9 & 17,5 & 35,4 & 36,9 & 33,5 \\
\hline Aveia & 3 & Adiantada & 38,8 & 39,6 & 38,2 & 35,0 & 38,7 & 32,6 & 22,0 & 17,6 & 26,1 \\
\hline Aveia & 3 & Atrasada & 35,6 & 37,6 & 32,6 & 55,8 & 57,5 & 53,0 & - & - & - \\
\hline Aveia & 6 & Radial & 21,0 & 17,0 & 20,1 & 12,9 & 14,1 & 13,7 & 21,2 & 15,9 & 25,7 \\
\hline
\end{tabular}

C, Sistema contínuo; AE, Sistema alternado esquerdo; AD, Sistema alternado direito.

O aumento da vazão e do adiantamento da posição das aletas possibilita maior capacidade de campo operacional ao conjunto mecanizado, pela possibilidade de operar com maior largura útil de aplicação e maior velocidade de deslocamento, tanto para o calcário como para a aveia, preservando os padrões de uniformidade de distribuição transversal.

\section{CONCLUSÃO}

As velocidades média e alta da esteira transportadora para a distribuição de calcário e a velocidade baixa para a distribuição de aveia fornecem uma ampla e suficiente faixa de dosagens com a variação de abertura da comporta dosadora. O aumento da vazão e do adiantamento da posição das aletas possibilita maior capacidade de campo operacional ao conjunto mecanizado, pela possibilidade de operar com maior largura útil de aplicação e maior velocidade de deslocamento. A largura máxima recomendada de aplicação foi de 13,5 metros para o calcário e para a aveia.

\section{REFERÊNCIAS}

ASAE. American Society of Agricultural Engineers. ASAE S341.2. Procedure for measuring distribution uniformity and calibrating granular broadcast spreaders. St Joseph: ASAE Standards, 1995. 3p.

BERNACKI, H.; KANAFOJSKI, C. Agricultural machines, theory and constructions. Poland: USDA/NSF, 1972. V.1, chapt.12, p.883.

DALLMEYER, A.U. Desenvolvimento de um rotor cônico para distribuição de calcário seco. 1985. 103f. Dissertação (Mestrado em Engenharia Agrícola) - Universidade Federal de Santa Maria, Santa Maria.
International Standard Organization. ISO 5690. Equipment for distributing fertilizers - Test methods - Part 1: full width fertlizers distributors. Geneve: ISO Standars handboook 13, Agricultural machinery, 1982. p.373-385.

MÁRQUEZ, L. Maquinaria para la preparación del suelo, la implantación de los cultivos y la fertilización. Madrid: Blake y Helsey, 2001. 496p.

MIALHE, L.G. Máquinas agrícolas: ensaios e certificação. Piracicaba, SP: Fundação de Estudos Agrários Luiz de Queiroz, 1996. 722p.

MOLIN, J.P. et al. Programa computacional para análise de distribuição transversal em aplicadores de fertilizantes e corretivos à lanço. In: CONGRESSO BRASILEIRO DE ENGENHARIA AGRÍCOLA, 21.; SIMPOSIO DE ENGENHARIA AGRÍCOLA DO CONE SUL, 1992, Santa Maria. Anais... Santa Maria: Sociedade Brasileira de Engenharia Agrícola, 1992. V.4, p.2097-2104.

ORTIZ-CAÑAVATE, J.; HERnÁNZ, J.L. Tecnica de la mecanizacion agraria. Madrid: Mundi-Prensa, 1989. 654p.

SILVA, P.E.H. Capacidade de trabalho e uniformidade de distribuição de dois distribuidores centrífugos de fertilizantes. 1982. 182f. Dissertação (Mestrado em Engenharia Agrícola) - Universidade Federal de Santa Maria.

SOUZA, J.M. Desempenho de um distribuidor centrífugo de disco na semeadura de arroz. 1984. 101f . Dissertação (Mestrado e m Engenharia Agrícola) - Universidade Federal de Santa Maria.

SRIVASTAVA, A.K. et al. Engineering principles of agricultural machines. Michigan: American Society of Agricultural Engineers, 1993. 601p.

VALDEZ, E. Determinacion y estudio de los perfiles de distribuicion de superfosfato, hiperfosfato y urea granulados, aplicados com la fertilizadora centrifuga. 2A-E-400. Agrinter, Montevidéo, v.14, n.4, p.1-42, 1978.

WEISS, A. Desenvolvimento de um distribuidor helicoidal para calcário seco. 1986. 79f. Dissertação (Mestrado em Engenharia Agrícola) - Universidade Federal de Santa Maria.

Ciência Rural, v.38, n.7, out, 2008. 